\title{
Design of a Multi-band, Dual Substrate Concentric Annular Ring Antenna
}

\author{
Gregory Mitchell and Amir Zaghloul \\ U.S. Army Research Laboratory \\ Adelphi, MD, USA \\ gregory.a.mitchell1.civ@mail.mil / amirz@vt.edu
}

\begin{abstract}
We present a multi-band and dual-polarization microstrip antenna designed for the $S$ - and $\mathrm{X}$-bands. The S-band element is an annular ring shorted with vias on the inner edge to suppress surface waves. The $\mathrm{X}$-band element is a combination of a concentric annular ring slot shorted on the outer edge and a narrowband patch. All elements achieve circular polarization by truncating opposing corners of the rings. Both bands utilize two orthogonal feeds to allow either right hand or left hand circular polarization. This paper shows performance optimized for the $\mathrm{X}$-band element at $7.8 \mathrm{GHz}$ and $9.2 \mathrm{GHz}$. We achieve a peak realized gain to boresight of $7.0 \mathrm{~dB}$ and $3.5 \mathrm{~dB}$ respectively.
\end{abstract}

Keywords-multi-band antenna, dual-polarization, dualsubstrate, concentric annular ring, microstrip

\section{INTRODUCTION}

Recent interest in multi-band radar for military applications necessitates the investigation of multi-band antenna approaches. Currently, several radar functions can only be obtained by a suite of different antennas that are challenging and costly to maintain, and difficult to network for realizing multi-mission capabilities. A single multi-band radar could replace current technology. The low profile and light weight designs of [1-4] show great promise for multi-band radar applications. The antenna geometry consists of a shorted annular ring for the low frequency element and a concentric annular ring slot for the high frequency element.

We propose utilizing two substrates allowing us to shrink the footprint of the S-band element. We optimize the radiating elements of the antenna for S-band and X-band respectively. Each of these elements achieves both right hand circular polarization (RHCP) and left hand circular polarization (LHCP). We investigate the propagation and radiation effects of the X-band element. The S-band characteristics at $3.5 \mathrm{GHz}$ are presented in a previous paper by the authors [5]. All models and simulations are performed using the finite difference time domain (FDTD) solver of CST Studio Suite 2015 .

\section{Multi-BAND ANTENNA}

We base the design of the antenna on the concentric shorted annular ring slot geometry first proposed by Dorsey and Zaghloul [1-3]. Fig. 1 shows the geometry of this antenna on a single substrate, and Fig. 2 shows the dimensions of the same antenna on a dual substrate, where $L_{1}=12.4 \mathrm{~mm}, L_{2}=9.7 \mathrm{~mm}$ and $\Delta T=3.7 \mathrm{~mm}$.

Truncating opposing corners of the two elements, by $\Delta T$ and $\Delta H$ respectively, achieves both RHCP and LHCP from a single excitation. Exciting one stripline feed, see Fig. 3, achieves RHCP while the other achieves LHCP at X-band. For each element, we terminate the additional ports with a $50 \Omega$ load. The quadrature needed for $\mathrm{CP}$ arises due to the different hypotenuse lengths between the opposing corners of the annular ring [6].

We use the shorting vias shown in Fig. 1 to cancel surface waves and suppress the parallel plate mode generated by the coupled stripline feed for the X-band element.

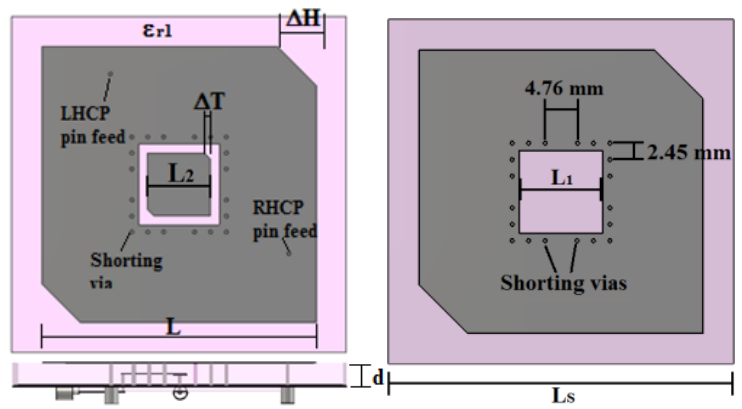

Fig. 1. Geometry of the concentric annular ring on a single substrate [5].
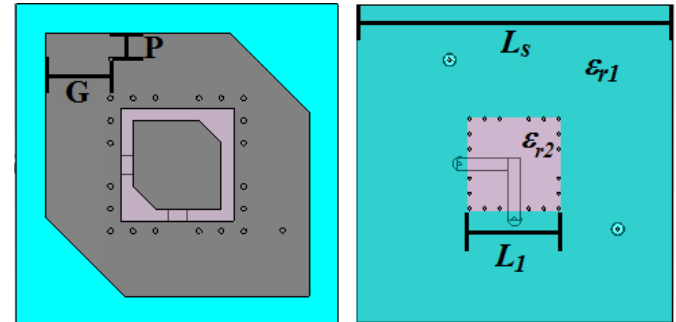

Fig. 2. Top-side antenna geometry (left) with substrate dimensions (right) [5].

TABLE I. ANTENNA DIMENSIONS IN MILLIMETERS

\begin{tabular}{|c|c|c|c|c|c|c|c|}
\hline $\boldsymbol{L}$ & $\boldsymbol{L}_{\boldsymbol{S}}$ & $\boldsymbol{\Delta H}$ & $\boldsymbol{P}$ & $\boldsymbol{G}$ & $\boldsymbol{\varepsilon}_{\boldsymbol{r} 1}$ & $\boldsymbol{\varepsilon}_{\boldsymbol{r} 2}$ & $\boldsymbol{d}$ \\
\hline 28.9 & 35.2 & 8.7 & 2.3 & 7.2 & 2.3 & 5.0 & 4.2 \\
\hline
\end{tabular}




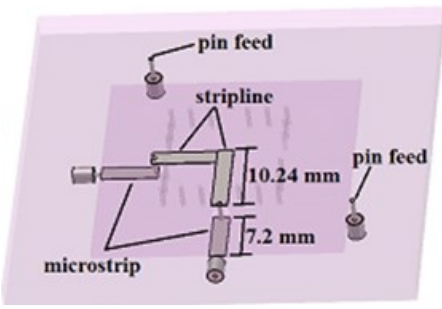

Fig. 3. Four-port feed network [5]. Pin feeds excite the S-band element and stripline feeds excite the X-band element.

The bottom left of Fig. 1 shows a 5 layer stack of a top metal layer, a Rogers RT5870 substrate, a metal ground layer, a Rogers RT5870 microstrip substrate, and a bottom microstrip transmission line metal layer. All metal layers are $0.1 \mathrm{~mm}$ thick. The top Rogers RT5870 substrate is $3.15 \mathrm{~mm}$ thick, and the lower microstrip substrate is $0.76 \mathrm{~mm}$ thick. The total profile of the antenna is $d=4.2 \mathrm{~mm}$. Fig 3. shows a $50 \Omega$ microstrip transmission line shorted to a $50 \Omega$ stripline transmission line. The stripline feed couples to the X-band annular ring slot and the X-band patch. There is a $0.1 \mathrm{~mm}$ separation between the orthogonal stripline feeds in the normal direction.

\section{X-BAND Simulation Results}

We show the return loss $\left(S_{11}\right)$ and axial ratio of the antenna at X-band in Fig. 4 and Fig. 5 respectively. The excitation of a single stripline yields a good match and $\mathrm{CP}$ axial ratio at 7.6 $\mathrm{GHz}$ and 9.2 GHz respectively. A minimum axial ratio of 3.0 is required for $\mathrm{CP}$. Fig. 6 shows the polar realized gain patterns and axial ratio at $7.6 \mathrm{GHz}$, while Fig. 7 shows the same at $9.2 \mathrm{GHz}$. We see a peak realized gain of $7.02 \mathrm{~dB}$ and a $3 \mathrm{~dB}$ beam width of $80^{\circ}$ at $7.6 \mathrm{GHz}$. We also see about a $60^{\circ}$ beam width of an axial ratio of 3.0 or better, although it is not centered to boresight. Furthermore, we see a peak realized gain of $3.52 \mathrm{~dB}$ and a $3 \mathrm{~dB}$ beam width of $60^{\circ}$ at $9.2 \mathrm{GHz}$. However, the beam width of the axial ratio is severely limited to only about $15^{\circ}$ before we exceed an axial ratio of 3.0. We attribute this performance to the fact that we optimized the feed for performance at 7.6 GHz as opposed to $9.2 \mathrm{GHz}$.

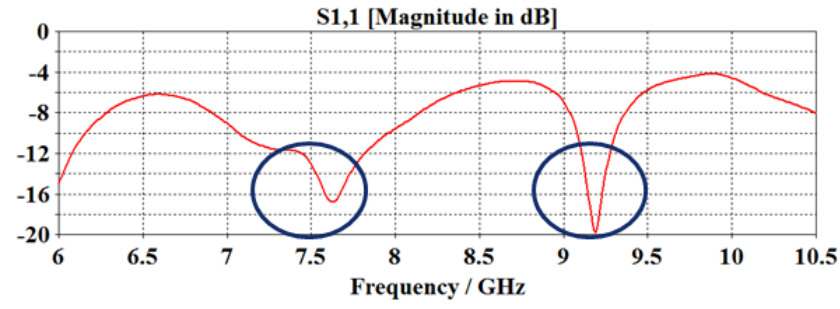

Fig. 4. Return loss of the X-band stripline excitations in Fig. 3.

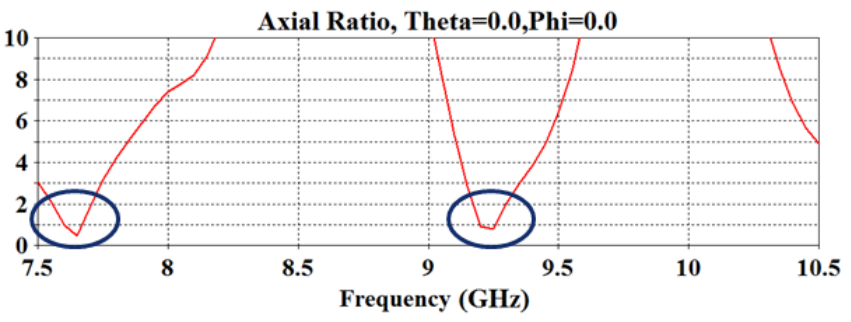

Fig. 5. Axial ratio to boresight of $\mathrm{X}$-band stripline excitations in Fig. 3.

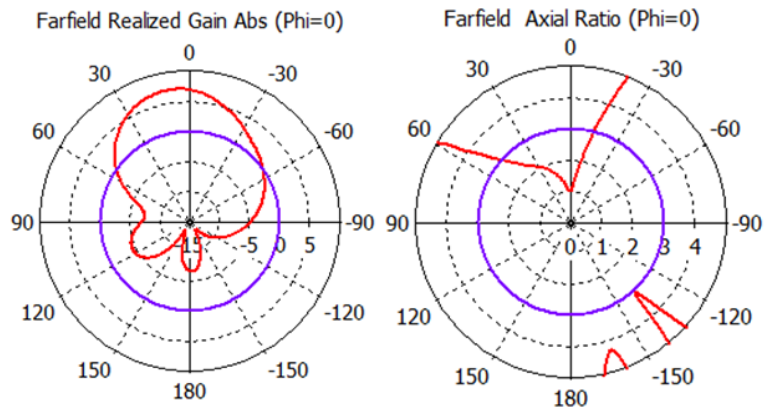

Fig. 6. Realized gain and axial ratio at $7.6 \mathrm{GHz}$.

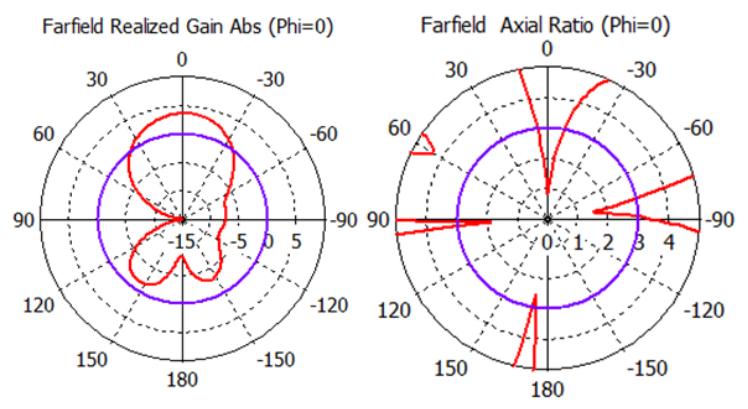

Fig. 7. Realized gain and axial ratio at 7.6 GHz.

\section{CONCLUSIONS}

We present a dual band, dual polarization planar antenna that operates at S- and X-band. By tapering opposite corners of both an annular ring and concentric annular ring slot, we see an axial ratio of better than 3.0 with good realized gain patterns to boresight at $7.6 \mathrm{GHz}$ and $9.2 \mathrm{GHz}$. We see that the return loss is better than $S_{11}=-16.0 \mathrm{~dB}$ at both frequencies. The beam width and gain of the $7.6 \mathrm{GHz}$ element exceeds that of the $9.2 \mathrm{GHz}$ element significantly. We believe that this is due to a weaker coupling of the stripline feed to the $9.2 \mathrm{GHz}$ element. Further optimization of the feed network at $9.2 \mathrm{GHz}$ could improve the performance,; however, an accompanying degradation of performance at $7.6 \mathrm{GHz}$ would be expected.

\section{REFERENCES}

[1] W. Dorsey and A. Zaghloul, "Dual-polarized dual-band antenna element for ISM bands", Proc. IEEE Ant. and Propagat. Society Int. Symp., APS/URSI, June 2009.

[2] A. Zaghloul and W. Dorsey, "Evolutionary development of a dual-band, dual-polarization, low-profile printed circuit antenna", Proc. Int. Conf. Electromag. in Advan. Appl., ICEAA, pp. 994-997, Sept. 2009.

[3] W. Dorsey and A. Zaghloul, "Dual-band dual-circularly pPolarized antenna element," IET Microw. and Ant. Propagat., vol. 7, pp. 283-290, 2013.

[4] M. Khan, Z. Yang, and K. Warnick, "Dual-circular-polarized highefficiency antenna for ku-band satellite communication", IEEE Ant. and Wirel. Propagat. Lett., vol. 13, pp. 1624-1627, 2014.

[5] G. Mitchell and A. Zaghloul, "Reduced Footprint of a Multi-band, Dual Polarization Microstrip Antenna", 32nd International Review of Progress in Applied Computational Electromagnetics (ACES), vol. 32, Feb. 2016.

[6] W. Chen, C. Wu, and K. Wong, "Single-feed square-ring microstrip antenna with truncated corners for compact circular polarisation Operation", Electr. Lett., vol. 34, iss. 11, pp. 1045-1047, 1998. 